contribution of the verderers and gives them certain additional powers. Provision was also made to enable the verderers to authorize the enclosure of additional land up to a maximum of five thousand acres, for the purpose of growing timber, and the enclosure of a maximum of three thousand acres for cultivation and improvement of grazing. The long-standing problem of the ancient and ornamental woods was met by providing that the verderers may authorize the Commissioners, subject to certain conditions, to make enclosures, individually not exceeding twenty acres in area, for the rehabilitation of these woods.

\section{RADIO-WAVE PROPAGATION AT VERY HIGH FREQUENCIES}

$T$

HE radio communication engineer is continually seeking opportunities of using the shorter wavelength portions of the radio spectrum for services and applications of various types. Reference was made recently in Nature (April 21, p. 617) to the fact that the report of the Beveridge Committee on broadcasting in Great Britain directed attention to the need for actively developing the use of fre. quencies above $30 \mathrm{Mc} / \mathrm{s}$. (wave-lengths shorter than $10 \mathrm{~m}$.) in order to relieve the congestion in Europe in the medium- and long-wave bands, and to provide a greater diversity in the broadcasting programmes available in Great Britain. The present British television service is being developed in the frequency range 4l-68 Mc./s., while a band of frequencies in the region of $90 \mathrm{Mc}$./s. is available for the development of sound broadcasting.

It was thus very timely that, at a meeting of the Radio Section of the Institution of Electrical Engineers held on April 11, three papers were read describing the results of recent investigations on the propagation of waves at certain very high frequencies. The first of these, entitled "A V.H.F. Field-Strength Survey on 90 Mc./s.", by H. L. Kirke, R. A. Rowden and $G$. I. Ross, describes measurements made by staff of the British Broadcasting Corporation on the radiation from an experimental transmitter at Wrotham in Kent. Automatic field-strength recording equipment was installed in a vehicle which cruised at a speed of about $1 \mathrm{~m}$.p.h. over a distance of from 50 to $500 \mathrm{yd}$. in the neighbourhood of each point selected for observation. In this manner measurements were made in various directions from the transmitter up to distances of some 60 miles, which includes the whole of the London area and a considerable portion of south-east England.

It was found that, while the field-strength varied markedly with small changes in position in a built-up area or along a tree-lined road, useful and consistent results could be obtained from the averages of the recorded readings. Thus, it was shown that buildings of normal height in London reduce the average received field by 10-12 db.; but the average for one street may be taken as the average for the district to an accuracy of about $\pm 4 \mathrm{db}$. The variation of field-strength along the street is less with horizontal than with vertical polarization. In hilly country, the minimum field-strength occurred on the near-side slope of a valley and not at the lowest point. On rising ground facing the transmitter, horizontally polarized waves gave stronger fields than vertical polarization; but, when once the peak of the hill has been passed, there is a range on the far-side slope in which vertically polarized radiation gives the stronger field, a fact which confirms the results of previous investigators. From all the results obtained in this work a predicted field-strength contour map has been prepared and is included in the paper. This map refers to the power $(25 \mathrm{~kW}$.) and aerial height (500 ft.) of a B.B.C. transmitting station now in experimental use at Wrotham. This investigation has shown that when the Wrotham station is brought into full operation, it is likely to gave a satisfactory broadcasting service, defined by a field-strength of $2 \mathrm{mV} . / \mathrm{m}$. or more, to Greater London and most of south-east England.

The other two papers read at the meeting were under the common title of "The Propagation of Metre Radio Waves beyond the Normal Horizon" and describe some theoretical and experimental investigations carried out during the past few years by staff of the Radio Division of the National Physical Laboratory. In Part 1, Dr. J. A. Saxton considers the propagation of very-high-frequency waves over land, and discusses the relative importance of changes in atmospheric refraction near the surface of the earth and the reflexion of waves from highlevel inversion layers. Using information on typical atmospheric conditions at various heights, obtained from the usual meteorological charts, the author presents some useful sets of graphs showing the field-strength to be expected at various distances up to $400 \mathrm{~km}$. (250 miles) from the transmitter. The comparative influence on the received field of the three main factors-simple atmospheric refraction, guiding of the waves in a low-level duct, and reflexion from inversion layers-is well illustrated by these graphs. Consideration is also given to the effects of scattering of radio energy by turbulent eddies in the atmosphere; but, except at distances exceeding about $250 \mathrm{~km}$., it appears that such scattering is of less importance than the effect of the mechanisms of abnormal refraction, which may be deemed to include reflexion at elevated inversion layers.

The third paper, forming Part 2 of the joint contribution, is entitled "Experimental Investigations at Frequencies of 90 and $45 \mathrm{Mc}$./s.", by Dr. J. A. Saxton, G. W. Luscombe and G. H. Bazzard. This describes the results of measurements of the fieldstrength on these frequencies over distances such that direct transmission from the sender to the receiver was impossible. Two paths, of lengths 110 and $270 \mathrm{~km}$., have been investigated on $90 \mathrm{Mc} . / \mathrm{s}$., and one of $160 \mathrm{~km}$. at $45 \mathrm{Mc}$. $/ \mathrm{s}$. The recorded fieldstrengths varied over a range of some $40 \mathrm{db}$., and there is some indication of a diurnal variation. The statistical distribution of quasi-peak field-strengths as a function of time has been determined, and it is shown that values of field considerably in excess of those corresponding to standard atmospheric refraction often occur. An attempt is made in the paper to interpret the observed field-strengths in terms of the meteorological data obtained from the routine radio sounding-balloon ascents made in Great Britain, and some degree of correlation has been obtained. The results of such investigations are of considerable importance in planning radio com. munication services for operation over distances well in excess of the normal optical range, and also in enabling an assessment to be made of the extent of any mutual interference likely to be experienced between broadcasting and other services operating in the same frequency channel. 